\title{
Fading Performance Evaluation of Adaptive MSER Beamforming Receiver for QAM Systems
}

\author{
Andrew Livingstone \\ Detica \\ Surrey Research Park \\ Guildford GU2 7YP, UK \\ E-mail: Andrew.livingstone@detica.com
}

\author{
Sheng Chen and Lajos Hanzo \\ School of Electronics and Computer Science \\ University of Southampton \\ Southampton SO17 1BJ, UK \\ E-mails: $\{$ sqc,lh $\} @$ ecs.soton.ac.uk
}

\begin{abstract}
The ever-increasing demand for mobile communication capacity has motivated the development of adaptive antenna array assisted spatial processing techniques for bandwidth efficiency, high-throughput quadrature amplitude modulation (QAM) systems. We evaluate performance of adaptive beamforming assisted detection for QAM systems in Rayleigh fading environments. An adaptive minimum symbol error rate design, referred to as the least symbol error rate, is shown to be capable of successfully operating in fast fading conditions and to consistently outperform the conventional adaptive beamforming benchmarker based on the least mean square algorithm.
\end{abstract}

\section{INTRODUCTION}

The ever-increasing demand for mobile communication capacity has motivated the development of adaptive antenna array assisted spatial processing techniques [1]-[10] in order to further improve the achievable spectral efficiency. A particular technique that has shown real promise in achieving substantial capacity enhancements is the use of adaptive beamforming with antenna arrays. Adaptive beamforming is capable of separating signals transmitted on the same carrier frequency, and thus provides a practical means of supporting multiusers in a space-division multiple-access scenario. Classically, the beamforming process is carried out by minimising the mean square error (MSE) between the desired output and the actual array output, and adaptive implementation of this minimum MSE (MMSE) design can be achieved using the well-known least mean square (LMS) algorithm [11],[12].

For a communication system, however, it is the bit error rate (BER) or symbol error rate (SER) that really matters. This has previously motivated the research in the adaptive beamforming design based on directly minimising the system's BER for binary phase shift keying modulation [13][18] and quadrature phase shift keying modulation system [19],[20]. For the sake of improving the achievable bandwidth efficiency, high-throughput quadrature amplitude modulation (QAM) schemes [21] have become popular in numerous wireless network standards. Recently, the adaptive minimum SER (MSER) beamforming design has been proposed for the QAM system [22] and its performance has been evaluated in stationary channel environments, in comparison with the classical adaptive MMSE beamforming design.

In this study, we concentrate on evaluating the fading performance of the adaptive MSER beamforming algorithm, referred to as the least symbol error rate (LSER) in [22]. Our simulation results confirm that the adaptive LSER-based beamforming is capable of successfully operating in fast fading conditions and it consistently outperforms the standard adaptive beamforming benchmarker based on the LMS algorithm.

\section{SYSTEM MODEL}

The system supports $S$ users, and each user transmits an $M$ QAM signal on the same carrier frequency of $\omega=2 \pi f$. For such a system, user separation can be achieved in the spatial or angular domain [8],[10] and the receiver is equipped with a linear antenna array consisting of $L$ uniformly spaced elements. Assume that the channel is narrow-band which does not induce intersymbol interference. Then the symbol-rate received signal samples can be expressed as

$$
x_{l}(k)=\sum_{i=1}^{S} A_{i} b_{i}(k) e^{j \omega t_{l}\left(\theta_{i}\right)}+n_{l}(k)=\bar{x}_{l}(k)+n_{l}(k),
$$

for $1 \leq l \leq L$, where $t_{l}\left(\theta_{i}\right)$ is the relative time delay at array element $l$ for source $i$ with $\theta_{i}$ being the direction of arrival for source $i, n_{l}(k)$ is a complex-valued Gaussian white noise with $E\left[\left|n_{l}(k)\right|^{2}\right]=2 \sigma_{n}^{2}, A_{i}$ is the narrow-band channel coefficient for user $i$, and $b_{i}(k)$ is the $k$ th symbol of user $i$ which takes the value from the $M$-QAM symbol set

$$
\mathcal{B} \triangleq\left\{b_{l, q}=u_{l}+j u_{q}, 1 \leq l, q \leq \sqrt{M}\right\}
$$

with $\Re\left[b_{l, q}\right]=u_{l}=2 l-\sqrt{M}-1$ and $\Im\left[b_{l, q}\right]=u_{q}=2 q-$ $\sqrt{M}-1$. Assume that source 1 is the desired user and the rest of the sources are interfering users. The desired-user signalto-noise ratio (SNR) is given by $\mathrm{SNR}=\left|A_{1}\right|^{2} \sigma_{b}^{2} / 2 \sigma_{n}^{2}$ and the desired signal-to-interferer $i$ ratio (SIR) is $\operatorname{SIR}_{i}=A_{1}^{2} / A_{i}^{2}$, for $2 \leq i \leq S$, where $\sigma_{b}^{2}$ denotes the $M$-QAM symbol energy. The received signal vector $\mathbf{x}(k)=\left[x_{1}(k) x_{2}(k) \cdots x_{L}(k)\right]^{T}$ can be expressed as

$$
\mathbf{x}(k)=\mathbf{P b}(k)+\mathbf{n}(k)=\overline{\mathbf{x}}(k)+\mathbf{n}(k),
$$

where $\mathbf{n}(k)=\left[n_{1}(k) n_{2}(k) \cdots n_{L}(k)\right]^{T}$, the system matrix $\mathbf{P}=\left[\begin{array}{llll}A_{1} \mathbf{s}_{1} & A_{2} \mathbf{s}_{2} \cdots A_{S} \mathbf{s}_{S}\end{array}\right]$ with the steering vector for source $i$ given by $\mathbf{s}_{i}=\left[e^{j \omega t_{1}\left(\theta_{i}\right)} e^{j \omega t_{2}\left(\theta_{i}\right)} \cdots e^{j \omega t_{L}\left(\theta_{i}\right)}\right]^{T}$, and the transmitted symbol vector $\mathbf{b}(k)=\left[b_{1}(k) b_{2}(k) \cdots b_{S}(k)\right]^{T}$. 


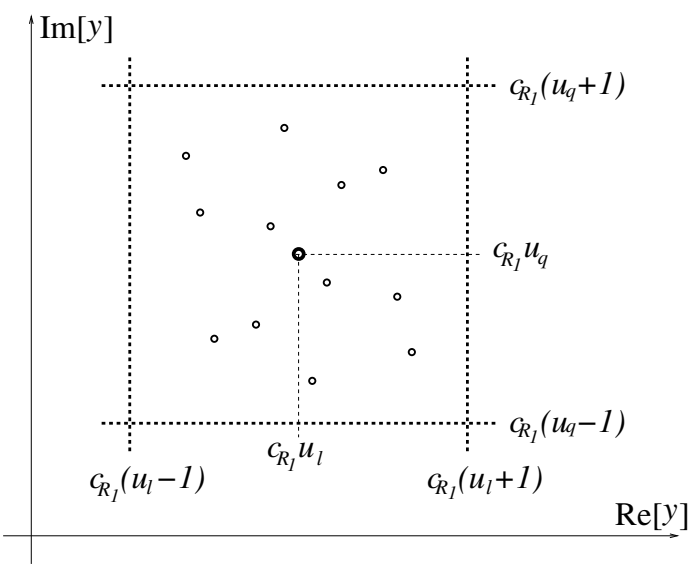

Fig. 1. Decision thresholds associated with point $c_{1} b_{l, q}$ assuming $c_{R_{1}}>0$ and $c_{I_{1}}=0$, and illustrations of symmetric distribution of $\mathcal{Y}_{l, q}$ around $c_{1} b_{l, q}$.

A beamformer is employed, whose soft output is given by

$$
y(k)=\mathbf{w}^{H} \mathbf{x}(k)=\mathbf{w}^{H}(\overline{\mathbf{x}}(k)+\mathbf{n}(k))=\bar{y}(k)+e(k)
$$

where $\mathbf{w}=\left[\begin{array}{ll}w_{1} & w_{2} \cdots w_{L}\end{array}\right]^{T}$ is the beamformer weight vector and $e(k)$ is Gaussian distributed with zero mean and $E\left[|e(k)|^{2}\right]=2 \sigma_{n}^{2} \mathbf{w}^{H} \mathbf{w}$. Define the combined system impulse

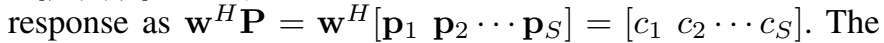
beamformer's output can alternatively be expressed as

$$
y(k)=c_{1} b_{1}(k)+\sum_{i=2}^{S} c_{i} b_{i}(k)+e(k)
$$

where the first term in the righthand side of equation is the desired user signal and the second term is the residual multiuser interference. Note that, in any detection scheme, the main tap $c_{1}$ must be known, that is the desired user's channel and associated steering vector, namely $\mathbf{p}_{1}=A_{1} \mathbf{s}_{1}$, must be known at the receiver. This fact is often overlooked. Provided that $c_{1}=c_{R_{1}}+j c_{I_{1}}$ satisfies $c_{R_{1}}>0$ and $c_{I_{1}}=0$, the symbol decision $\hat{b}_{1}(k)=\hat{b}_{R_{1}}(k)+j \hat{b}_{I_{1}}(k)$ can be made as

$$
\begin{aligned}
& \hat{b}_{R_{1}}(k)=\left\{\begin{aligned}
u_{1}, & \text { if } y_{R}(k) \leq c_{R_{1}}\left(u_{1}+1\right) \\
u_{l}, & \text { if } c_{R_{1}}\left(u_{l}-1\right)<y_{R}(k) \leq c_{R_{1}}\left(u_{l}+1\right) \\
u_{\sqrt{M},} & \text { if } y_{R}(k)>c_{R_{1}}\left(u_{\sqrt{M}}-1\right)
\end{aligned}\right. \\
& \hat{b}_{I_{1}}(k)=\left\{\begin{array}{cl}
u_{1}, & \text { if } y_{I}(k) \leq c_{R_{1}}\left(u_{1}+1\right) \\
u_{q}, & \text { if } c_{R_{1}}\left(u_{q}-1\right)<y_{I}(k) \leq c_{R_{1}}\left(u_{q}+1\right) \\
u_{\sqrt{M}}, & \text { if } y_{I}(k)>c_{R_{1}}\left(u_{\sqrt{M}}-1\right)
\end{array}\right.
\end{aligned}
$$

where $y(k)=y_{R}(k)+j y_{I}(k)$ and $\hat{b}_{1}(k)$ is the estimate for $b_{1}(k)=b_{R_{1}}(k)+j b_{I_{1}}(k)$. Fig. 1 depicts the decision thresholds associated with the decision $\hat{b}_{1}(k)=b_{l, q}$. In general, $c_{1}=\mathbf{w}^{H} \mathbf{p}_{1}$ is complex-valued and the operation

$$
\mathbf{w}^{\text {new }}=\frac{c_{1}^{\text {old }}}{\left|c_{1}^{\text {old }}\right|} \mathbf{w}^{\text {old }}
$$

can be used to make $c_{1}$ real and positive. This rotation operation is linear and it does not change the system's SER.

\section{ADAPTIVE MMSE BEAMFORMING}

The traditional design for the beamformer (4) is the MMSE solution, which can be implemented adaptively using the classical LMS algorithm [11],[12]. The MMSE beamforming design is computationally attractive, because it admits the closed-form solution given the second order statistics of the underlying system. Specifically, by minimising the MSE criterion $E\left[\left|b_{1}(k)-y(k)\right|^{2}\right]$, the MMSE solution for the beamformer's weight vector is given as

$$
\mathbf{w}_{\text {MMSE }}=\left(\mathbf{P P}^{H}+\frac{2 \sigma_{n}^{2}}{\sigma_{b}^{2}} \mathbf{I}_{L}\right)^{-1} \mathbf{p}_{1}
$$

where $\mathbf{I}_{L}$ denotes the $L \times L$ identity matrix. To adaptively implement the MMSE solution, the unknown second-order statistics can be estimated based on a block of training data. Furthermore, by considering a single-sample "estimate" of the MSE, the stochastic adaptive LMS algorithm is derived as

$$
\begin{gathered}
\tilde{\mathbf{w}}(k+1)=\hat{\mathbf{w}}(k)+\mu\left(b_{1}(k)-y(k)\right)^{*} \mathbf{x}(k), \\
\hat{c}_{1}(k+1)=\tilde{\mathbf{w}}^{H}(k+1) \hat{\mathbf{p}}_{1}, \\
\hat{\mathbf{w}}(k+1)=\frac{\hat{c}_{1}(k+1)}{\left|\hat{c}_{1}(k+1)\right|} \tilde{\mathbf{w}}(k+1),
\end{gathered}
$$

where $y(k)=\hat{\mathbf{w}}^{H}(k) \mathbf{x}(k), \hat{\mathbf{p}}_{1}$ is an estimated $\mathbf{p}_{1}$, and (11) and (12) implement the weight rotation operation. The step size $\mu$ is the algorithmic parameter that should be set appropriately in order to ensure an adequate performance in terms of convergence rate and steady-state MSE misadjustment.

\section{AdAPTIVE MSER BEAMFORMING}

However, since the SER is the true performance indicator, it is desired to consider the optimal MSER Beamforming solution. Denote the $N_{b}=M^{S}$ number of legitimate sequences of $\mathbf{b}(k)$ as $\mathbf{b}_{i}, 1 \leq i \leq N_{b}$. The noise-free part of the received signal $\overline{\mathbf{x}}(k)$ only takes values from the finite signal set defined by $\mathcal{X} \triangleq\left\{\overline{\mathbf{x}}_{i}=\mathbf{P b}_{i}, 1 \leq i \leq N_{b}\right\}$. The set $\mathcal{X}$ can be partitioned into $M$ subsets, depending on the value of $b_{1}(k)$ as $\mathcal{X}_{l, q} \triangleq$ $\left\{\overline{\mathbf{x}}_{i} \in \mathcal{X}: b_{1}(k)=b_{l, q}\right\}, 1 \leq l, q \leq \sqrt{M}$. Similarly the noise-free part of the beamformer's output $\bar{y}(k)$ only takes values from the scalar set $\mathcal{Y} \triangleq\left\{\bar{y}_{i}=\mathbf{w}^{H} \overline{\mathbf{x}}_{i}, 1 \leq i \leq N_{b}\right\}$, and $\mathcal{Y}$ can be divided into the $M$ subsets conditioned on $b_{1}(k)$

$$
\mathcal{Y}_{l, q} \triangleq\left\{\bar{y}_{i} \in \mathcal{Y}: b_{1}(k)=b_{l, q}\right\}, 1 \leq l, q \leq \sqrt{M} .
$$

The following two lemmas, summarising the properties of the signal subsets $\mathcal{Y}_{l, q}, 1 \leq l, q \leq \sqrt{M}$, are useful in the derivation of the SER expression for the beamformer (4).

Lemma 1: The subsets $\mathcal{Y}_{l, q}, 1 \leq l, q \leq \sqrt{M}$, satisfy the shifting properties

$$
\begin{gathered}
\mathcal{Y}_{l+1, q}=\mathcal{Y}_{l, q}+2 c_{1}, 1 \leq l \leq \sqrt{M}-1, \\
\mathcal{Y}_{l, q+1}=\mathcal{Y}_{l, q}+j 2 c_{1}, 1 \leq q \leq \sqrt{M}-1,
\end{gathered}
$$

$\mathcal{Y}_{l+1, q+1}=\mathcal{Y}_{l, q}+(2+j 2) c_{1}, 1 \leq l, q \leq \sqrt{M}-1$ 
Lemma 2: The points of $\mathcal{Y}_{l, q}$ are distributed symmetrically around the symbol point $c_{1} b_{l, q}$ with respect to the two horizontal decision boundaries and the two vertical decision boundaries that separate $\mathcal{Y}_{l, q}$ from the other subsets.

Lemma 2, as illustrated in Fig. 1, is a direct consequence of symmetric distribution of the symbol constellation (2).

For the beamformer with weight vector $\mathbf{w}$, denote

$$
\begin{aligned}
P_{E}(\mathbf{w}) & =\operatorname{Prob}\left\{\hat{b}_{1}(k) \neq b_{1}(k)\right\}, \\
P_{E_{R}}(\mathbf{w}) & =\operatorname{Prob}\left\{\hat{b}_{R_{1}}(k) \neq b_{R_{1}}(k)\right\}, \\
P_{E_{I}}(\mathbf{w}) & =\operatorname{Prob}\left\{\hat{b}_{I_{1}}(k) \neq b_{I_{1}}(k)\right\} .
\end{aligned}
$$

It is then easy to see that the SER is given by

$$
P_{E}(\mathbf{w})=P_{E_{R}}(\mathbf{w})+P_{E_{I}}(\mathbf{w})-P_{E_{R}}(\mathbf{w}) P_{E_{I}}(\mathbf{w}) .
$$

The conditional probability density function (PDF) of $y(k)$ given $b_{1}(k)=b_{l, q}$ is a Gaussian mixture defined by

$$
p\left(y \mid b_{l, q}\right)=\frac{1}{N_{s b} 2 \pi \sigma_{n}^{2} \mathbf{w}^{H} \mathbf{w}} \sum_{i=1}^{N_{s b}} e^{-\frac{\left|y-\bar{y}_{i}^{(l, q)}\right|^{2}}{2 \sigma_{n}^{2} \mathbf{w}^{H} \mathbf{w}}},
$$

where $N_{s b}=N_{b} / M$ is the size of $\mathcal{Y}_{l, q}, \bar{y}_{i}^{(l, q)}=\bar{y}_{R_{i}}^{(l, q)}+$ $j \bar{y}_{I_{i}}^{(l, q)} \in \mathcal{Y}_{l, q}$, and $y=y_{R}+j y_{I}$. Noting that $c_{1}$ is real and positive and taking into account the symmetric distribution of $\mathcal{Y}_{l, q}$ (lemma 2), for $2 \leq l \leq \sqrt{M}-1$, the conditional error probability of $\hat{b}_{R_{1}}(k) \neq u_{l}$ given $b_{R_{1}}(k)=u_{l}$ is

$$
P_{E_{R}, l}(\mathbf{w})=\frac{2}{N_{s b}} \sum_{i=1}^{N_{s b}} Q\left(g_{R_{i}}^{(l, q)}(\mathbf{w})\right),
$$

where

$$
Q(u)=\frac{1}{\sqrt{2 \pi}} \int_{u}^{\infty} e^{-\frac{z^{2}}{2}} d z
$$

and

$$
g_{R_{i}}^{(l, q)}(\mathbf{w})=\frac{\bar{y}_{R_{i}}^{(l, q)}-c_{R_{1}}\left(u_{l}-1\right)}{\sigma_{n} \sqrt{\mathbf{w}^{H} \mathbf{w}}} .
$$

Further taking into account the shifting property (lemma 1), it can be shown that

$$
P_{E_{R}}(\mathbf{w})=\gamma \frac{1}{N_{s b}} \sum_{i=1}^{N_{s b}} Q\left(g_{R_{i}}^{(l, q)}(\mathbf{w})\right),
$$

where $\gamma=\frac{2 \sqrt{M}-2}{\sqrt{M}}$. It is seen that $P_{E_{R}}$ can be evaluated using (real part of) any single subset $\mathcal{Y}_{l, q}$. Similarly, $P_{E_{I}}$ can be evaluated using (imaginary part of) any single $\mathcal{Y}_{l, q}$ as

$$
P_{E_{I}}(\mathbf{w})=\gamma \frac{1}{N_{s b}} \sum_{i=1}^{N_{s b}} Q\left(g_{I_{i}}^{(l, q)}(\mathbf{w})\right)
$$

with

$$
g_{I_{i}}^{(l, q)}(\mathbf{w})=\frac{\bar{y}_{I_{i}}^{(l, q)}-c_{R_{1}}\left(u_{q}-1\right)}{\sigma_{n} \sqrt{\mathbf{w}^{H} \mathbf{w}}} .
$$

Note that the SER is invariant to a positive scaling of $\mathbf{w}$. The MSER solution $\mathbf{w}_{\text {MSER }}$ is defined as the weight vector that minimises the upper bound of the SER given by

$$
P_{E_{B}}(\mathbf{w})=P_{E_{R}}(\mathbf{w})+P_{E_{I}}(\mathbf{w}),
$$

that is,

$$
\mathbf{w}_{\mathrm{MSER}}=\arg \min _{\mathbf{w}} P_{E_{B}}(\mathbf{w}) .
$$

The upper bound (28) is very tight, that is, very close to $P_{E}(\mathbf{w})$. Therefore, the solution obtained by minimising this upper bound is practically equivalent to that of minimising $P_{E}(\mathbf{w})$. Unlike the MMSE solution, the MSER solution does not admits a closed-form solution. However, the optimisation problem (29) can be solved using a gradient algorithm [22]. It is worth emphasising that there exist infinitely many MSER solutions which forms an infinite half line in the beamforming weight space. This helps numerical optimisation, as any point in this line is an MSER solution. In our experience, we have not found a case in which the optimisation algorithm converges to some local minima of the SER surface.

In practice, the system matrix $\mathbf{P}$ is unknown (except its first column). Therefore adaptive implementation is required to realise the MSER beamforming. The PDF $p(y)$ of $y(k)$ can be estimated using the Parzen window estimate [23]-[25] based on a block of training data. This leads to an estimated SER for the beamformer. Minimising this estimated SER based on a gradient optimisation yields an approximated MSER solution. To derive a sample-by-sample adaptive algorithm, consider a single-sample "estimate" of $p(y)$

$$
\tilde{p}(y, k)=\frac{1}{2 \pi \rho_{n}^{2}} e^{-\frac{|y-y(k)|^{2}}{2 \rho_{n}^{2}}}
$$

and the corresponding one-sample SER "estimate" $\tilde{P}_{E_{B}}(\mathbf{w}, k)$. Using the instantaneous stochastic gradient of $\nabla \tilde{P}_{E_{B}}(\mathbf{w}, k)=$ $\nabla \tilde{P}_{E_{R}}(\mathbf{w}, k)+\nabla \tilde{P}_{E_{I}}(\mathbf{w}, k)$ with

$$
\begin{aligned}
& \nabla \tilde{P}_{E_{R}}(\mathbf{w}, k)=\frac{\gamma}{2 \sqrt{2 \pi} \rho_{n}} e^{-\frac{\left(y_{R}(k)-\hat{c}_{R_{1}}(k)\left(b_{R_{1}}(k)-1\right)\right)^{2}}{2 \rho_{n}^{2}}} \\
& \times\left(-\mathbf{x}(k)+\left(b_{R_{1}}(k)-1\right) \hat{\mathbf{p}}_{1}\right)
\end{aligned}
$$

and

$$
\begin{aligned}
\nabla \tilde{P}_{E_{I}}(\mathbf{w}, k) & =\frac{\gamma}{2 \sqrt{2 \pi} \rho_{n}} e^{-\frac{\left(y_{I}(k)-\hat{c}_{R_{1}}(k)\left(b_{I_{1}}(k)-1\right)\right)^{2}}{2 \rho_{n}^{2}}} \\
\times & \left(j \mathbf{x}(k)+\left(b_{I_{1}}(k)-1\right) \hat{\mathbf{p}}_{1}\right)
\end{aligned}
$$

gives rise to the adaptive LSER algorithm [22]

$$
\begin{gathered}
\tilde{\mathbf{w}}(k+1)=\hat{\mathbf{w}}(k)+\mu\left(-\nabla \tilde{P}_{E_{B}}(\hat{\mathbf{w}}(k), k)\right), \\
\hat{c}_{1}(k+1)=\tilde{\mathbf{w}}^{H}(k+1) \hat{\mathbf{p}}_{1}, \\
\hat{\mathbf{w}}(k+1)=\frac{\hat{c}_{1}(k+1)}{\left|\hat{c}_{1}(k+1)\right|} \tilde{\mathbf{w}}(k+1),
\end{gathered}
$$

The step size $\mu$ and the kernel width $\rho_{n}$ are the two algorithmic parameters that should be set appropriately in order to ensure an adequate performance in terms of convergence rate and steady-state SER misadjustment. As emphasised in Section II, the column $\mathbf{p}_{1}$ of the system matrix associated with the desired user must be known in receiver. Usually, the steering vector $\mathbf{s}_{1}$ associated with the desired user is known at receiver. The desired user's channel $A_{1}$ can always be estimated accurately during training. Thus, in the following simulation study, we assume a perfect $\mathbf{p}_{\mathbf{1}}$ at receiver. 


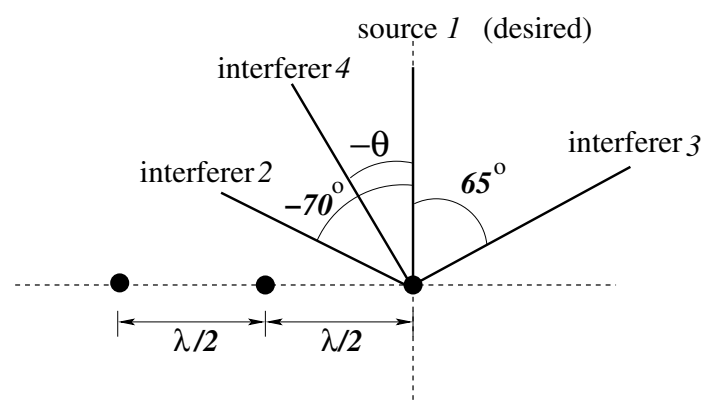

Fig. 2. Locations of the user sources with respect to the three-element linear array with $\lambda / 2$ element spacing, $\lambda$ being the wavelength, where $\theta<65^{\circ}$.

\section{Simulation Study}

The system consisted of four users and a three-element antenna array. Fig. 2 shows the locations of the four users graphically, where the angular separation between the desired user and the interfering user 4 was $\theta<65^{\circ}$.

Non-fading system. The modulation scheme was 16-QAM and all the channels $A_{i}, 1 \leq i \leq 4$, were time-invariant. With all the four users having an equal signal power, Figs. 3 to 5 compare the SER performance of the MSER beamforming solution to that of the MMSE beamforming solution under the conditions of a) the minimum anugular separation between the desired user 1 and the interfering user 4 being $\theta=32^{\circ}$, b) $\theta=30^{\circ}$ and c) $\theta=28^{\circ}$, respectively. For the case of equal user power with the minimum anugular separation $\theta=32^{\circ}$, the MSER beamforming solution had an SNR gain of $2 \mathrm{~dB}$ over the MMSE solution at the SER level of $10^{-3}$, as can be seen from Fig. 3. When the minimum anugular separation of the system was reduced to $\theta=30^{\circ}$, as depicted in Fig. 4, the SNR gain of the MSER beamformer over the MMSE one was increased to $4 \mathrm{~dB}$. With the minimum anugular separation further reduced to $\theta=28^{\circ}$, the MMSE beamforming solution became incapable of removing the interference and exhibited a high SER floor, as illustrated in Fig. 5. In contrast, the MSER beamformer remained capable of effectively removing the interference and achieving an adequate SER performance. A near-far situation was next simulated. Under the conditions

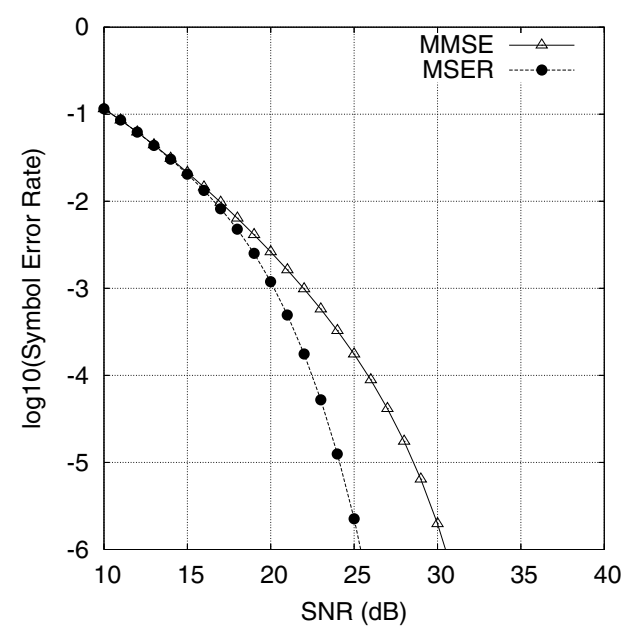

Fig. 3. Comparison of SER performance for the non-fading system with $\theta=32^{\circ}$ and $\operatorname{SIR}_{i}=0 \mathrm{~dB}$ for $2 \leq i \leq 4$.

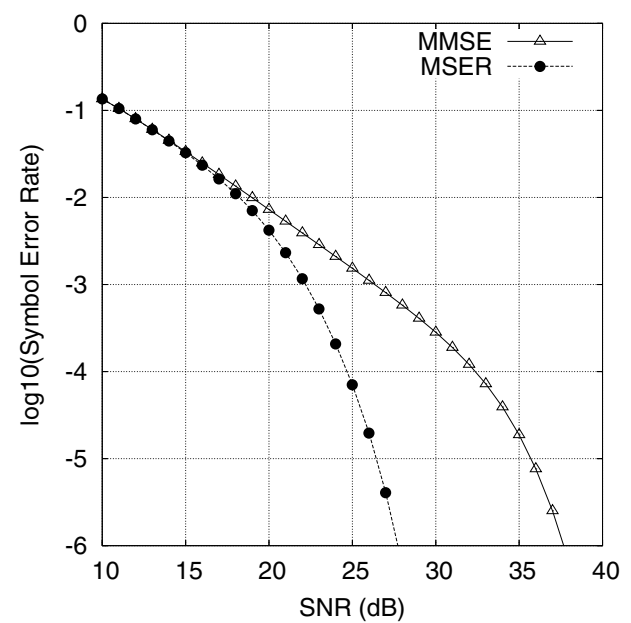

Fig. 4. Comparison of SER performance for the non-fading system with $\theta=30^{\circ}$ and $\operatorname{SIR}_{i}=0 \mathrm{~dB}$ for $2 \leq i \leq 4$.

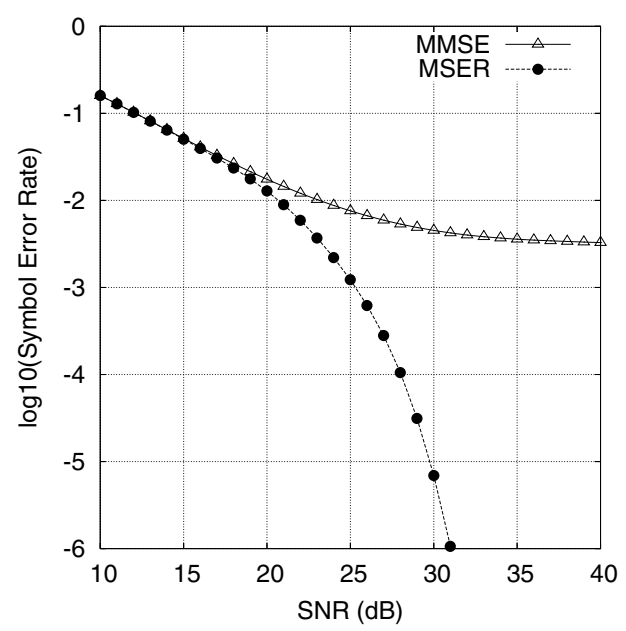

Fig. 5. Comparison of SER performance for the non-fading system with $\theta=28^{\circ}$ and $\mathrm{SIR}_{i}=0 \mathrm{~dB}$ for $2 \leq i \leq 4$.

that $\theta=30^{\circ}$, user 1 and user 2 had the same signal power but users 3 and 4 had $2 \mathrm{~dB}$ more power than users 1 and 2, Fig. 6 compares the performance of the two beamformers. By comparing Fig. 4 with Fig. 6 , it can be seen that, when facing stronger interfering users 3 and 4, the MMSE solution faltered while the MSER solution sufferred from very little degradation. This clearly demonstrated that the MSER beamformer is more robust in near-far situations than the MMSE beamformer. Lastly, the performance of both the LMS and LSER beamformers are compared with those of the theoretic MMSE and MSER solutions in Fig. 7 under the same conditions as those of Fig. 4. The superiority of the adaptive LSER beamformer over the adaptive LMS beamformer is clearly demonstrated in Fig. 7, where it can be seen that the performance of the adaptive LMS beamformer was notably deviated from its theoretic MMSE solution at high SNRs while the adaptive LSER beamformer closely followed its theoretic MSER solution even at high SNR values. Appropriate values for the step size $\mu$ as well as the kernel width $\rho_{n}$ were found empirically, and the SER performance presented in Fig. 7 for the two adaptive beamformers were calculated after 


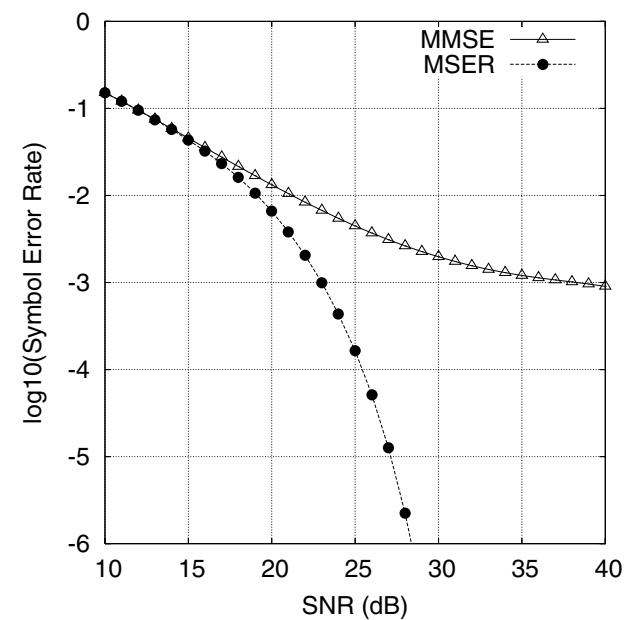

Fig. 6. Comparison of SER performance for the non-fading system with $\theta=30^{\circ}, \mathrm{SIR}_{2}=0 \mathrm{~dB}$ and $\mathrm{SIR}_{3}=\mathrm{SIR}_{4}=-2 \mathrm{~dB}$.

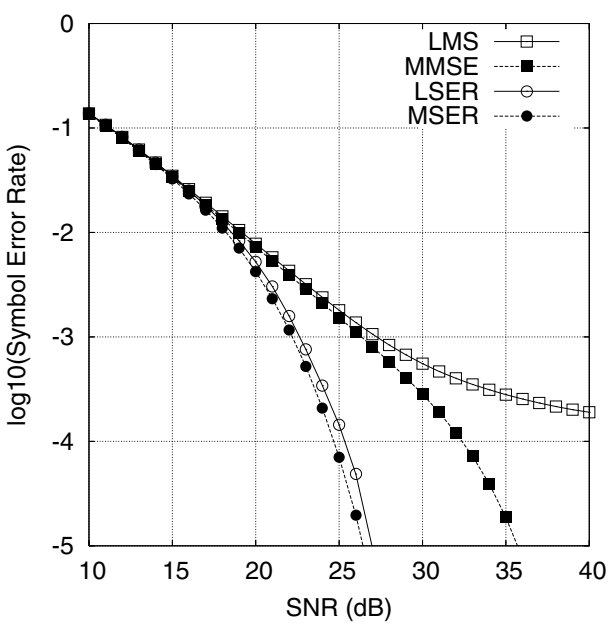

Fig. 7. SER performance comparsion of adaptive beamformers for the nonfading system with $\theta=30^{\circ}$ and $\mathrm{SIR}_{i}=0 \mathrm{~dB}$ for $2 \leq i \leq 4$.

convergence. Influence of $\mu$ and $\rho_{n}$ to the SER performance were further investigated in the following fading simulation.

Fading system. The modulation scheme was 64-QAM. Fading channels were simulated, where magnitudes of $A_{i}$ for $1 \leq$ $i \leq 4$ were Rayleigh processes with the normalised Doppler frequence $\bar{f}_{D}$ and each channel $A_{i}$ had the root mean power of $\sqrt{0.5}+j \sqrt{0.5}$. Thus the average $\operatorname{SIR}_{i}=0 \mathrm{~dB}$ for $2 \leq i \leq 4$. Continuously fluctuating fading was used, which provided a different fading magnitude and phase for each transmitted symbol. The transmission frame structure consisted of 50 training symbols followed by 450 data symbols. Decision-directed adaptation was employed during data transmission, in which the adaptive beamforming detector's decision $\hat{b}_{1}(k)$ was used to substitute for $b_{1}(k)$. The SER of an adaptive beamforming detector was calculated based on Monte Carlo simulation using at least $2 \times 10^{5}$ frames, depending on the value of $\bar{f}_{D}$. Two initialisations were used for the LSER algorithm, where the initial weight vector $\mathbf{w}(0)$ was set to either $\mathbf{w}_{\text {MMSE }}$ of the initial channel condition or $[0.1+j 0.00 .1+j 0.00 .1+j 0.0]^{T}$, and the performance were observed to be very similar for these two initialisations.

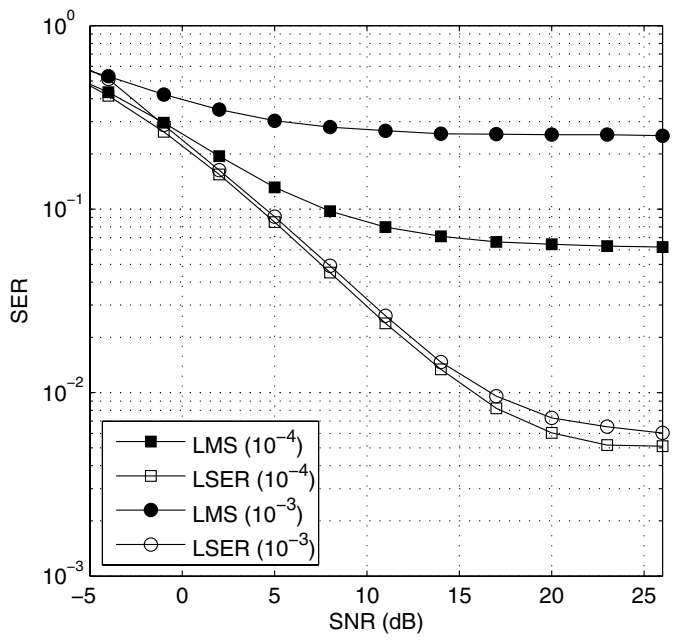

Fig. 8. Comparison of SER performance for the two normalised Doppler frequencies $\bar{f}_{D}=10^{-4}$ and $10^{-3}$ with the minimum angular separation $\theta=27^{\circ}$. The LMS algorithm has a step size $\mu=0.0002$, while the LSER algorithm has a step size $\mu=0.00005$ and a kernel width $\rho_{n}=4 \sigma_{n}$.

Given the minimum angular separation $\theta=27^{\circ}$, Fig. 8 compares the SER of the adaptive LSER beamformer with that of the LMS-based one, for the two normalised Doppler frequencies $\bar{f}_{D}=10^{-4}$ and $10^{-3}$. It can be seen from Fig. 8 that the SER performance of the LSER beamformer degraded only slightly when the fading rate increased from $\bar{f}_{D}=10^{-4}$ to $10^{-3}$. This demonstrates that the LSER algorithm has an excellent tracking ability, capable of operating in fast fading conditions. We next investigated the influence of the adaptive algorithm's parameters. Given $\bar{f}_{D}=10^{-4}$, Fig. 9 show the influence of the adaptive algorithm's parameters, $\mu$ for the LMS, and $\mu$ and $\rho_{n}$ for the LSER, on the SER performance for a low average SNR value of $15 \mathrm{~dB}$, while Fig. 10 depicts the results for a high average SNR value of $30 \mathrm{~dB}$. These results also explain how we came to use $\mu=0.0002$ for the LMS and $\mu=0.00005$ and $\rho_{n}=4 \sigma_{n}$ for the LSER in the simulation. Lastly, given $\bar{f}_{D}=10^{-3}$, we varied the minimum angular separation $\theta$ uniformly in $\left[20^{\circ}, 50^{\circ}\right]$ and averaged the SER performance. The results are plotted in Fig. 11.

\section{CONCLUSIONS}

An adaptive MSER beamforming technique has been studied for multiple antenna aided multiuser communication systems with QAM signalling. An adaptive implementation of the MSER beamforming solution, referred to as the LSER technique, has been evaluated in the simulation. The results obtained in this study clearly show that the adaptive LSER beamforming is capable of operating successfully in fast fading conditions and it consistently outperforms the adaptive LMS beamforming benchmarker.

\section{ACKNOWLEDGEMENTS}

The financial support of the EU under the auspice of the Newcom project is gratefully acknowledged. 


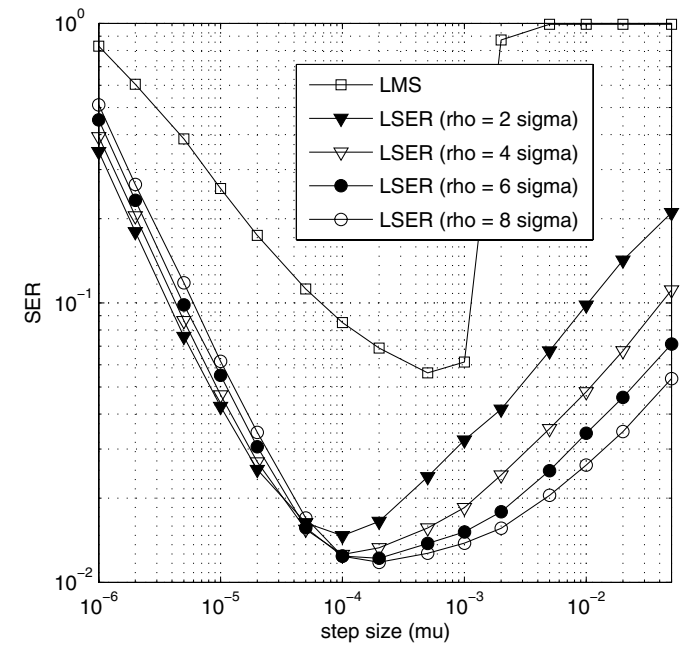

Fig. 9. Influence of the adaptive algorithm's parameters to the SER performance, given $\theta=27^{\circ}, \bar{f}_{D}=10^{-4}$ and average $\mathrm{SNR}=15 \mathrm{~dB}$.

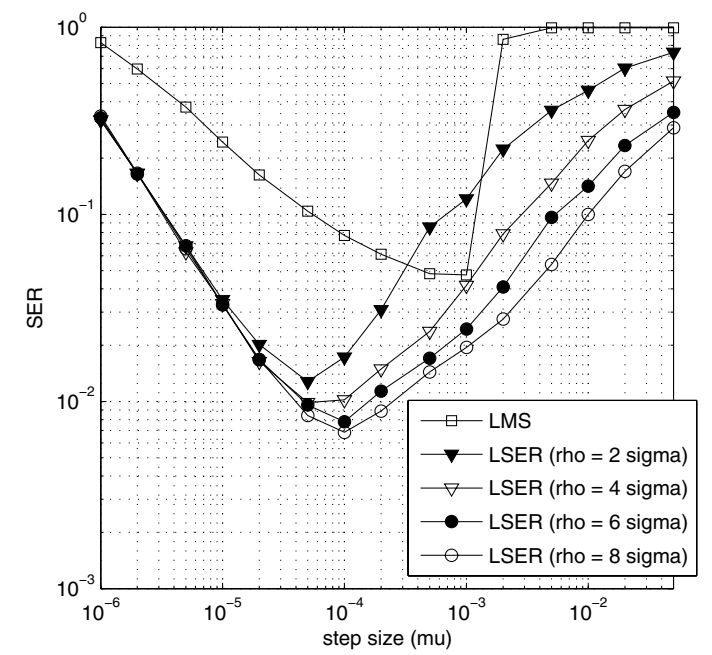

Fig. 10. Influence of the adaptive algorithm's parameters to the SER performance, given $\theta=27^{\circ}, \bar{f}_{D}=10^{-4}$ and average $\mathrm{SNR}=30 \mathrm{~dB}$.

\section{REFERENCES}

[1] J.H. Winters, J. Salz and R.D. Gitlin, "The impact of antenna diversity on the capacity of wireless communication systems," IEEE Trans. Communications, Vol.42, No.2, pp.1740-1751, 1994.

[2] J. Litva and T. K.Y. Lo, Digital Beamforming in Wireless Communications. London: Artech House, 1996.

[3] L. C. Godara, "Applications of antenna arrays to mobile communications, Part I: Performance improvement, feasibility, and system considerations," Proc. IEEE, Vol.85, No.7, pp.1031-1060, 1997.

[4] R. Kohno, "Spatial and temporal communication theory using adaptive antenna array," IEEE Personal Communications, Vol.5, No.1, pp.28-35, 1998.

[5] J.H. Winters, "Smart antennas for wireless systems," IEEE Personal Communications, Vol.5, No.1, pp.23-27, 1998.

[6] P. Vandenameele, L. van Der Perre and M. Engels, Space Division Multiple Access for Wireless Local Area Networks. Boston: Kluwer Academic Publishers, 2001.

[7] J.S. Blogh and L. Hanzo, Third Generation Systems and Intelligent Wireless Networking - Smart Antenna and Adaptive Modulation. Chichester: John Wiley, 2002.

[8] A. Paulraj, R. Nabar and D. Gore, Introduction to Space-Time Wireless Communications. Cambridge: Cambridge University Press, 2003.

[9] A.J. Paulraj, D.A. Gore, R.U. Nabar and H. Bölcskei, "An overview of MIMO communications - A key to gigabit wireless," Proc. IEEE, Vol.92, No.2, pp.198-218, 2004.

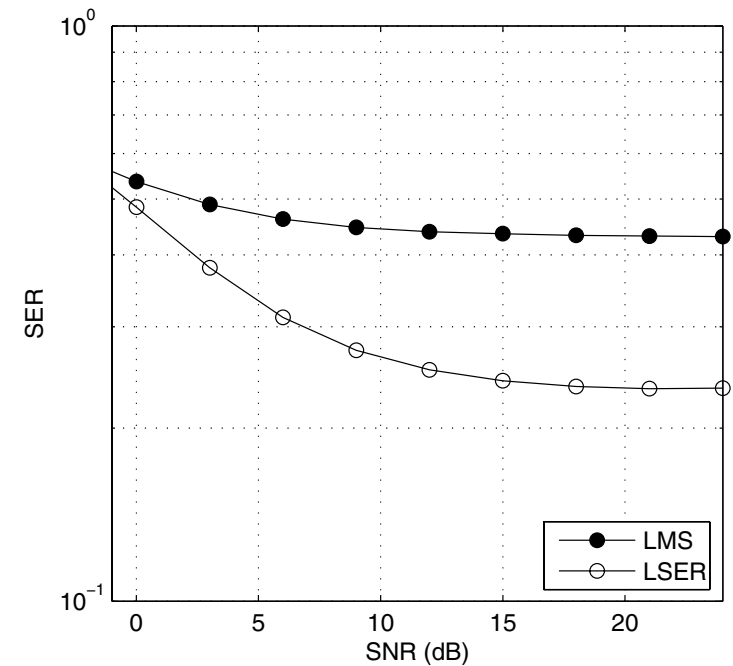

Fig. 11. Comparison of average SER performance for the normalised Doppler frequency $\bar{f}_{D}=10^{-3}$ with the minimum angular separation $\theta$ uniformly distributed in $\left[20^{\circ}, 50^{\circ}\right]$. The LMS algorithm has a step size $\mu=0.0002$, while the LSER algorithm has a step size $\mu=0.00005$ and a kernel width $\rho_{n}=4 \sigma_{n}$

[10] D. Tse, and P. Viswanath, Fundamentals of Wireless Communication. Cambridge, U.K.: Cambridge University Press, 2005.

[11] B. Widrow and S.D. Stearns, Adaptive Signal Processing. Englewood Cliffs, NJ: Prentice-Hall, 1985.

[12] S. Haykin, Adaptive Filter Theory. 3rd edition, Upper Saddle River, NJ: Prentice-Hall, 1996.

[13] S. Chen, L. Hanzo and N.N. Ahmad, "Adaptive minimum bit error rate beamforming assisted receiver for wireless communications," in Proc. ICASSP 2003 (Hong Kong, China), April 6-10, 2003, vol.IV, pp.640643.

[14] A. Wolfgang, N.N. Ahmad, S. Chen and L. Hanzo, "Genetic algorithm assisted minimum bit error rate beamforming," in Proc. VTC 2004Spring, May 17-19, 2004, pp.142-146.

[15] I.D.S. Garcia, J.J.S. Marciano, Jr., and R.D. Cajote, "Normalized adaptive minimum bit-error-rate beamformers," in Proc. IEEE Region 10 TENCON Conf., Nov. 21-24, 2004, vol.2, pp.625-628.

[16] Y.-H. Liu and Y.-H. Yang, "Adaptive minimum bit error rate multitarget array algorithm," in Proc. IEEE 6th CAS Symp. Emerging Technologies: Frontiers of Mobile and Wireless Communication, 31 May-2 Jun, 2004, vol.2, pp.745-748.

[17] S. Chen, N.N. Ahmad and L. Hanzo, "Adaptive minimum bit error rate beamforming," IEEE Trans. Wireless Communications, vol.4, no.2, pp.341-348, 2005

[18] L.-Y. Fan, H.-B. Zhang and H. Chen, "Minimum bit error rate beamforming for pre-FFT OFDM adaptive antenna array," in Proc. VTC 2005 Fall, Sept.25-28, 2005, vol.6, pp.359-363.

[19] S. Chen, L. Hanzo, N.N. Ahmad and A. Wolfgang, "Adaptive minimum bit error rate beamforming assisted QPSK receiver," in Proc. ICC 2004, 2004, Vol.6, pp.3389-3393.

[20] S. Chen, L. Hanzo, N.N. Ahmad and A. Wolfgang, "Adaptive minimum bit error rate beamforming assisted receiver for QPSK wireless communication," Digital Signal Processing, vol.15, no.6, pp.545-567, 2005 .

[21] L. Hanzo, S.X. Ng, T. Keller and W. Webb, Quadrature Amplitude Modulation: From Basics to Adaptive Trellis-Coded, Turbo-Equalised and Space-Time Coded OFDM, CDMA and MC-CDMA Systems. John Wiley and IEEE Press: Chichester, UK, 2004.

[22] S. Chen, H.-Q. Du and L. Hanzo, "Adaptive minimum symbol error rate beamforming assisted receiver for quadrature amplitude modulation systems," in Proc. VTC2006-Spring (Melbourne, Australia), May 7-10, 2006, CDROM 5 pages.

[23] E. Parzen, "On estimation of a probability density function and mode," The Annals of Mathematical Statistics, Vol.33, pp.1066-1076, 1962.

[24] B.W. Silverman, Density Estimation. London: Chapman Hall, 1996.

[25] A.W. Bowman and A. Azzalini, Applied Smoothing Techniques for Data Analysis. Oxford: Oxford University Press, 1997. 